\title{
Therapeutic Strategies in HCC: Radiation Modalities
}

\author{
R. Gallicchio, ${ }^{1}$ A. Nardelli, ${ }^{2}$ P. Mainenti, ${ }^{2}$ A. Nappi, ${ }^{1}$ D. Capacchione, ${ }^{1}$ V. Simeon, ${ }^{1}$ \\ C. Sirignano, ${ }^{2}$ F. Abbruzzi, ${ }^{3}$ F. Barbato, ${ }^{4}$ M. Landriscina, ${ }^{1,5}$ and G. Storto ${ }^{1}$ \\ ${ }^{1}$ Nuclear Medicine Department, Istituto di Ricovero e Cura a Carattere Scientifico (IRCCS), \\ Centro di Riferimento Oncologico di Basilicata (CROB), Via P. Pio 1, 85028 Rionero in Vulture, Italy \\ ${ }^{2}$ Istituto di Biostrutture e Bioimmagini, Consiglio Nazionale delle Ricerche, 80145 Napoli, Italy \\ ${ }^{3}$ Oncology Unit, IRCCS Giovanni Paolo II, 70124 Bari, Italy \\ ${ }^{4}$ CMO Oplonti Medical Centre, 80058 Torre Annunziata, Italy \\ ${ }^{5}$ Oncology Unit, Università degli Studi di Foggia, 71122 Foggia, Italy \\ Correspondence should be addressed to G. Storto; giosto24@hotmail.com
}

Received 28 February 2016; Revised 24 May 2016; Accepted 5 June 2016

Academic Editor: Tapas Das

Copyright (C) 2016 R. Gallicchio et al. This is an open access article distributed under the Creative Commons Attribution License, which permits unrestricted use, distribution, and reproduction in any medium, provided the original work is properly cited.

Patients with hepatocellular carcinoma (HCC) comply with an advanced disease and are not eligible for radical therapy. In this distressed scenario new treatment options hold great promise; among them transarterial chemoembolization (TACE) and transarterial metabolic radiotherapy (TAMR) have shown efficacy in terms of both tumor shrinking and survival. External radiation therapy (RTx) by using novel three-dimensional conformal radiotherapy has also been used for HCC patients with encouraging results while its role had been limited in the past for the low tolerance of surrounding healthy liver. The rationale of TAMR derives from the idea of delivering exceptional radiation dose locally to the tumor, with cell killing intent, while preserving normal liver from undue exposition and minimizing systemic irradiation. Since the therapeutic efficacy of TACE is being continuously disputed, the TAMR with ${ }^{131} \mathrm{I}$ Lipiodol or ${ }^{90} \mathrm{Y}$ microspheres has gained consideration providing adequate therapeutic responses regardless of few toxicities. The implementation of novel radioisotopes and technological innovations in the field of RTx constitutes an intriguing field of research with important translational aspects. Moreover, the combination of different therapeutic approaches including chemotherapy offers captivating perspectives. We present the role of the radiation-based therapies in hepatocellular carcinoma patients who are not entitled for radical treatment.

\section{Introduction}

Hepatocellular carcinoma (HCC) is among the most common tumors worldwide and although most cases still occur in developing countries in South-East Asia and Africa, its incidence is also increasing in developed industrialized states [1-3]. Almost 700,000 new cases are diagnosed yearly throughout the world and unfortunately the prognosis remains poor since more than 500,000 deaths are ascribed to HCC each year [2]. Where the incidence of HCC is rising this datum seems to be related to the better diagnostics and healthcare of patients complying with cirrhosis and the significant increase in chronic hepatitis C. Noteworthy, a significantly higher mortality related to HCC hepatitis- and cirrhosis-based disease is expected in women in next years
[4-6]. Contamination of foodstuffs by aflatoxins in some areas cannot also be excluded [7-9]. In this context, only a limited number of cases are eligible for decisive treatments, such as resection, transplantation, ethanol injection, or radiofrequency ablation, since these curative approaches are also indeterminate. On the other hand, a plethora of palliative/alternative treatments has been recently proposed for patients who are not entitled for radical therapy or those who exhibit inoperable locoregional disease without distant metastases. Since in this setting systemic chemotherapy and the conventional external beam radiation therapy (RTx) have been demonstrated ineffective in terms of both survival and safety, transarterial chemoembolization with Lipiodol (an oily medium; TACE) and the transarterial metabolic radiation therapy (TAMR) have proven useful $[10,11]$. 
However, the therapeutic efficacy of chemoembolization is being continuously disputed, both in retrospective and randomized studies [12, 13]. Also, this approach has been demonstrated to provide a similar overall survival when compared to transarterial ethanol ablation, but with a worse complete tumor response, a shorter time to progression, and a shorter progression free survival. Moreover, TACE is associated or not with eluting drugs and seems to not offer significant advantages in comparison with a wait and watch approach [14-17]. Lastly, only few data support the value of the concurrent intra-arterial chemotherapy with external beam radiotherapy (CCRT) when compared to TACE alone [18]. This distressed scenario has endorsed the use of metabolic radiotherapy via hepatic artery administration by labeling Lipiodol with several radioisotopes [19-23] and using Yttrium-90 microspheres or radiolabeled antibodies as valuable tools to attain adequate therapeutic response $[24,25]$. The rationale of TAMR derives from the idea of delivering exceptional radiation dose locally to the tumor, with cell killing intent, while preserving healthy liver and the rest of organs from undue exposition. Moreover, the dual vascularization of the liver represents an added value when treating hypervascularized neoplasms such as HCC whose hematic support is mainly provided from the hepatic artery. Among others, Lipiodol has been labeled with ${ }^{131}$ I since 1964 [26] and, more recently, implemented in the management of HCC as well with ${ }^{188} \operatorname{Re}[21,27]$. Both radioisotopes have been used in humans showing variable response rates. Recently, ${ }^{90} \mathrm{Y}$ microsphere therapy has been implemented in preliminary randomized studies with encouraging results in terms of target lesion response as well as overall survival, despite some toxicities $[28,29]$. Recent technological advances in the field of RTx, such as the three-dimensional conformal definition of the target volume, make it suitable, alone or in combination, for patients complying with inoperable HCC [30].

\section{Basic Pathophysiology and Rationale of Radiation Modalities}

The pathophysiology of HCC is a multifactorial event. Inflammation, necrosis, fibrosis, and continuing regeneration characterize both chronic liver disease and cirrhotic liver and may contribute to HCC development in a crescendo. However, dysplastic and regenerative nodules found in cirrhotic livers do not necessarily sustain a progression to HCC, depending on the kinds of proliferating cells (smallcell dysplastic nodules increase risk more than large-cell nodules). Although hepatitis B virus (HBV) was initially supposed to be the primum movens, subsequent studies failed to identify $\mathrm{HBV}$ infection as the main independent risk factor [31]. In most cases HCC develops in patients with underlying cirrhotic liver disease of various etiologies, including patients whose $\mathrm{HBV}$ infection markers are negative [32]. On the other hand, in patients with hepatitis $C$ virus, HCC frequently occurs in the setting of cirrhosis. The transformation process also encompasses a multitude of pathways that can be modified by external and environmental factors. Finally, primary genetic changes may be the cause of delayed apoptosis and increased cellular proliferation but also result from hepatocarcinogenetic factors (Figure 1). From a radiotherapeutic point of view, radiation delivery to liver lesions is significantly limited by the tolerance of surrounding normal liver parenchyma. Although the underlying cause of hepatic radiation toxicity is a venocclusive disease, the ultimate damage and the risk of developing complication depend on how functional subunits (acini in liver) of organs are structured. Liver is a typical example of a radiobiologically parallel architecture model. As a result, the increased risk of complication depends on dose distribution throughout the whole organ and the exceeding patients' functional reserve rather than the maximum dose to a limited area. Unlike external radiation therapy, internal radiotherapy may convey radioisotopes (and radiation) either percutaneously or through transarterial approach acting as radioembolization. In particular biodistribution and metabolic studies in TAMR have shown that radiopharmaceuticals are fixed in the liver, tumors, and lungs (arteriopulmonary hepatic shunts) the unbound amount (quote; quantity) is eliminated mainly through the urinary tract (30-50\% of injected activity) [33]. The rationale of TAMR derives from the idea of delivering exceptional radiation dose locally to the tumor, with cell killing intent, while preserving healthy liver and the rest of organs from undue exposition. Moreover, the dual vascularization of the liver represents an added value when treating hypervascularized neoplasms such as HCC whose hematic support is mainly provided from the hepatic artery. In fact, the hepatic artery supplies $30 \%$ of the blood flow to the normal liver parenchyma but greater than $90 \%$ to hepatic tumors.

\section{The Role of Ionizing Radiations in the Management of HCC}

Transarterial metabolic radiotherapy plays a crucial role in the management of patients with primary and metastatic liver malignancies. In particular, the efficacy of these locoregional radiation-based treatments has been demonstrated in HCC, which is recognized to be a systemic chemoresistant cancer, making treatment of these tumors widely approved in the field of interventional radiooncology. This kind of internal radiotherapy is based on the locoregional delivery of radioisotopes either percutaneously or throughout transarterial approach. The ${ }^{131}$ I labeled Lipiodol was the first implemented agent and attempts to use it in humans were performed since 1986 [34]. Lipiodol is an oily medium based substance which has been shown to be selectively retained in tumor when administered intra-arterially and had been originally used as a contrast agent for diagnosing HCC [35]. Even if the mechanism of retention of Lipiodol within the HCC environment is not completely understood, it seems related to the different arteriolar density between the hypervascular HCC and the normal liver parenchyma, to the accumulation into peritumoral sinusoids, to the penetration and slower wash-out from HCC cells [36]. Coupling Lipiodol with the ${ }^{131}$ I radiohalogen has yielded a suitable radiopharmaceutical for treating HCC with emission of beta particles 


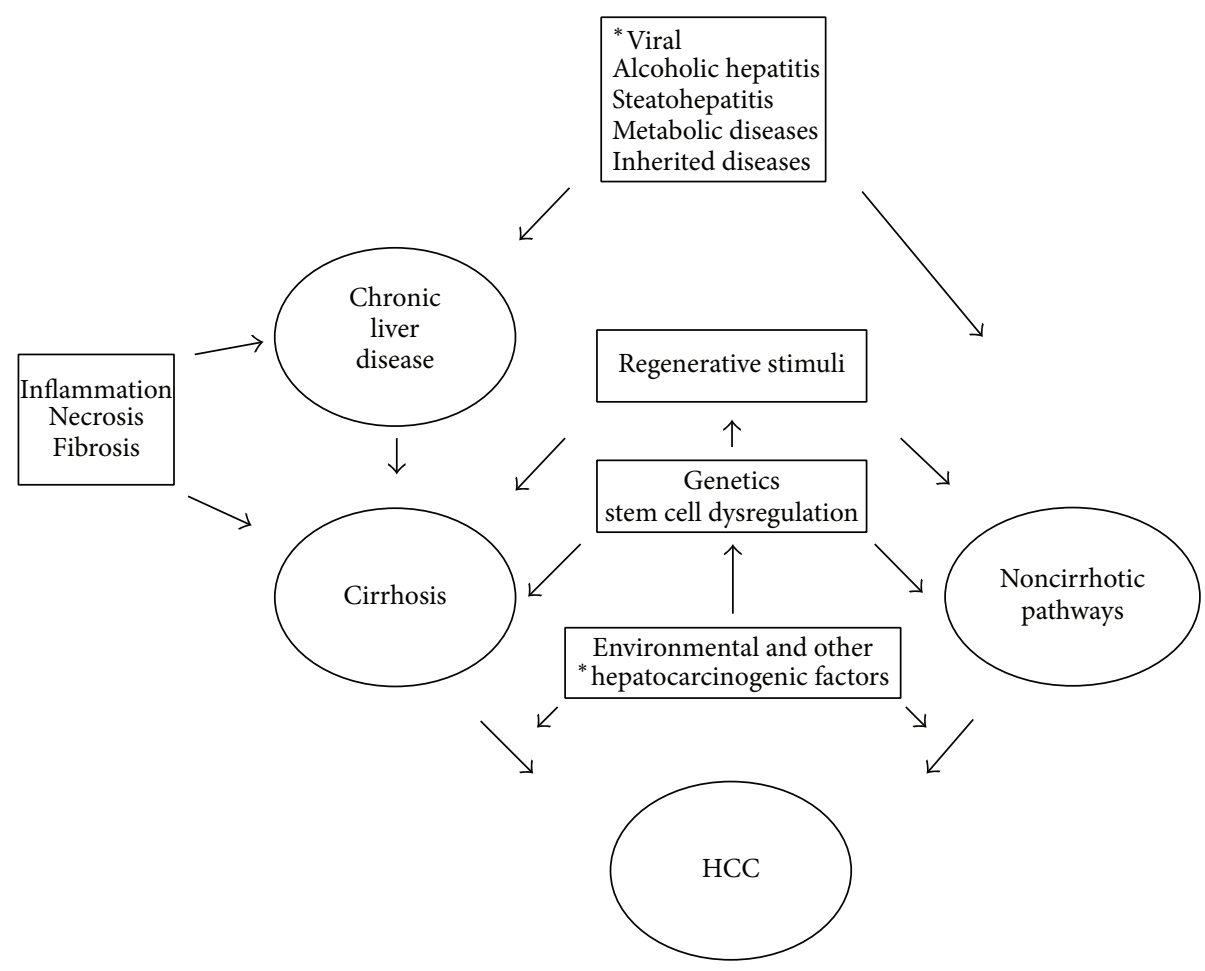

FIGURE 1: Basic pathophysiology of hepatocellular carcinoma (HCC). Viral, infections causing chronic hepatitis (type B, type C, type D, and others). Metabolic diseases, that is, alpha-1-antitrypsin deficiency. Inherited diseases, that is, Wilson disease and hemochromatosis. Environmental, that is, aflatoxins. $*$ : related to.

$(606 \mathrm{keV})$, despite some drawbacks (see radioprotection) due to the gamma-ray emission ( $364 \mathrm{keV} ; 82 \%$ abundance) and the long half-life (8.05 days). However, the gamma-ray emission allows dosimetric estimates. From the beginning this agent has shown a favourable tumor/nontumor liver ratio and several studies have been performed to demonstrate its biodistribution in nontarget organs as well. Reported values of tumor/nontumor ratios are between 4.3 and 20 allowing the delivery of a significant amount of irradiation to the tumor. Moreover, the retention of ${ }^{131} \mathrm{I}$ Lipiodol shows an inverse correlation with the tumor size and its effective halflife was reported to be approximately 4.6 days [37, 38]. Alternatively, both ${ }^{188} \mathrm{Re}$ Lipiodol (beta emission $2.1 \mathrm{MeV}$, half-life of 16.9 hours, and presence of gamma emission $155 \mathrm{keV}$ energy, suitable for dosimetry) and ${ }^{90} \mathrm{Y}$ Lipiodol (beta emission $2.2 \mathrm{MeV}$, half-life of 2.67 days, and tissue range penetration of $2.5 \mathrm{~mm}$ ) have also been used because of the favourable characteristics of these radioelements [39, 40], but some studies have been limited to animals or failed to demonstrate adequate safety and efficacy (Table 1) [41, 42].

Recently, microspheres labeled with ${ }^{90} \mathrm{Y}$ have been used in several clinical studies with encouraging rates of response. The development of the microspheres composed of resin or glass containing this radioelement in a stable form avoided the toxicity problems encountered at the beginning due to the systematic release of the radionuclide [43-45]. The favourable radiochemical and therapeutic characteristics of ${ }^{90} \mathrm{Y}$, especially when using high activity levels [46], have resulted in the increasing use of this agent similar to use of ${ }^{90} \mathrm{Y}$ for radioimmunotherapy of lymphoma [47].

Although a standardized RTx does not exist for liver, technological advances in the field of treatment planning have recently provided the means for delivering appropriate killing doses to a defined liver lesion, achieving satisfactory therapeutic ratios [48]. In this way, complications became acceptable. These novel irradiation techniques include the three-dimensional conformal radiotherapy for the accurate definition of the HCC target volume as well as of surrounding organs [49]. Treatment plans use multiple fields to irradiate the tumor and spare normal tissues. The above-mentioned RTx has been used for cirrhotic HCC patients not eligible for radical therapies, with encouraging results [50,51]. Moreover, some studies have indicated that stereotactic body radiation therapy is a safe and effective modality treatment for HCC [52-57]. This new therapeutic approach has gained an outstanding importance since it can be a bridge modality in patients who are candidate for transplantation $[58,59]$.

\section{Indications, Contraindication, and Special Precautions}

According to the pharmacokinetics of radio-coupled Lipiodol and microspheres, they can be properly indicated in patients having HCC with portal vein thrombosis [60, 61]. Such types of therapies can be also be employed as adjuvant 
TABLE 1: Examples of radioisotopes used for HCC transarterial metabolic radiotherapy.

\begin{tabular}{lcccc}
\hline Radioelements & Half-life (days) & Maximum beta energy $(\mathrm{MeV})$ & Maximum range in tissues $(\mathrm{mm})$ & $\mathrm{Gamma}$ energy $(\mathrm{KeV})$ \\
\hline Iodine-131 & 8.05 & 6.06 & 2 & 364 \\
Rhenium-186 & 3.7 & 1.7 & 10 & 137 \\
Rhenium-188 & 0.7 & 2.1 & 12 & 155 \\
Yttrium-90 & 2.67 & 2.2 & 8.7 & None \\
Holmium-166 & 1.1 & 1.85 & & 80.6 \\
\hline
\end{tabular}

treatment near the surgical resection, reducing the risk of recurrence [62]. However, this agent seems to be more effective when it is used in a neoadjuvant approach, since surgery can provoke the release of growth factors while neighbouring synchronous metastases could be comprised in the therapeutic plan [63]. Additional indications are the palliative treatment of not operable HCC, though it should be preferentially implemented in patients without distant metastases.

In general, the treatment should be reserved to HCC nodules less than $6-8 \mathrm{~cm}$ in diameter. It could be implemented as curative therapy of small-size HCC lesions which are inaccessible or as neoadjuvant treatment before hepatic transplantation in order to reduce the risk of recurrence on the graft. Finally, the two other indications can be evoked for TAMR and include the reduction of tumor burden in case of HCC that are too large to be operated and as antalgic treatment for patients who suffer from hyperalgesic HCC not more responsive to conventional analgesia. Concerning the external radiation approach, the patients could be eligible in case of HCC (either primary or recurrent) when surgery or percutaneous ablative therapies are unfeasible and difficult or patients demonstrate a refusal. In addition, patients presenting the longest tumor diameter of $\leq 5.0 \mathrm{~cm}$ and those exhibiting Child-Turcotte-Pugh Class A or B (no severely compromised liver function) can be considered. In particular, external beam radiotherapy can be safely considered for patients with 1-3 lesions and sufficient uninvolved liver parenchyma [64-67].

Contraindications of TAMR include the presence of distant metastases and patients complying with evolved cirrhosis or locally very advanced HCC and/or the presence of comorbidities as severe respiratory or renal insufficiency as well as bone marrow suppression (to be evaluated also in case of external radiation therapy). Moreover, patients with a documented pathology of the aorta or femoral arteries with iodine allergy and pregnancy status cannot be considered for this therapy. Finally, other contraindications embrace patients who had previous RTx to the liver or those treated with capecitabine within the two previous months. Patients who are scheduled to receive capecitabine at any time following treatment with ${ }^{90} \mathrm{Y}$ resin microspheres and those having greater than $20 \%$ lung shunting of the hepatic artery blood flow cannot be enrolled for TAMR. The lung represents a critical organ in the implementation of transarterial metabolic radiotherapy since pulmonary uptake rises in reason of a secondary release of the Lipiodol from the liver or in presence of arteriopulmonary hepatic shunt. In the case of ${ }^{90} \mathrm{Y}$ microspheres, the excessive irradiation may result in radiation hepatitis. An inadvertent delivery of microspheres to the gallbladder or to the gastrointestinal tract or pancreas can effect, respectively, a cholecystitis and pancreatitis. Also, a high level of implanted radiation and/or excessive shunting to the lung may lead to radiation pneumonitis. In the course of radiation therapy, asthenia, anorexia, and transient hepatalgies rise with temperature and a brief alteration of hepatic tests and leucopoenia should be considered, whereas prolonged fever, severe hepatic insufficiency, and gastroenteric haemorrhages are serious but rare side effects (as shown below).

Contraindications from commonly used ionizing radiation-based therapies (internal and external) for HCC are the following:

(i) Distant metastases (others than liver).

(ii) Evolved cirrhosis or locally very advanced HCC.

(iii) Severe respiratory or renal insufficiency.

(iv) Bone marrow suppression.

(v) Documented pathology of the aorta or femoral arteries (internal).

(vi) Iodine allergy ( ${ }^{131}$ I Lipiodol).

(vii) Pregnancy status.

(viii) Previous external beam radiation therapy to the liver.

(ix) Previous treatment with capecitabine within the two previous months or treatment with capecitabine at any time following treatment $\left({ }^{90} \mathrm{Y}\right.$ microspheres).

(x) Greater than 20\% lung shunting of the hepatic artery blood flow (internal).

\section{Administration and Treatment Sequence}

The two ways of administration that can be performed include selective injection into a hepatic artery via gastroduodenal artery or hyper-selective injection at the right- or lefthand side of the hepatic artery to the depth of the subsegmental arteries $[68,69]$. The last vascular access, once multiple foci have been excluded, allows an increase of delivered radiation dose to the tumor sparing normal liver and lungs. According to different studies, the administered activity generally ranges from 0.74 to $4.40 \mathrm{GBq}$ for ${ }^{131} \mathrm{I}$ Lipiodol and from 2.0 to $3.0 \mathrm{GBq}$ for ${ }^{90} \mathrm{Y}$ microspheres, respectively 
[70]. Therapeutic activities have also been settled on the basis of dosimetry and alternatively computed taking into account the dose delivered to the target or to the critical organs [71, 72]. On the other hand, the super-selective approach excludes the prophylactic irradiation of the healthy parenchyma that has been established effectively in reducing relapse or recurrence. From a methodological point of view, this approach requires a slow injection in case of small diameter artery in order to avoid reflux (flow rate to be used: 10-20 mL per hour).

The injection is carried out at the time of hepatic arteriography according to the technique of Seldinger [73]. The left femoral artery is the preferred access route in order to reduce operators exposure, even if standardized administration protocols do not exist. When ${ }^{90} \mathrm{Y}$ labeled microspheres are used, hepatic scintigraphy after the injection into the hepatic artery of macroaggregates of Tc-labeled human albumin is required. The scan allows evaluation and quantification of pulmonary shunts, if any, and of gastrointestinal shunts. It can also be used to estimate the dose to both the target and critical organs as well. Using ${ }^{131}$ I labeled Lipiodol, it is mandatory to hospitalize the patients in a protected room (see radioprotection) and to perform an abdominal scintigraphy searching for tumor retention and pulmonary uptake. Injection can be repeated every two-three months in patients presenting with large tumors and supplementary injections could be implemented when an objective response has been demonstrated. In general, the effectiveness of retreatment, if any, depends mainly on the amount of Lipiodol or microspheres retained by the tumor. Lastly, succeeding treatments can also be implemented in case of multiple foci with the intent to cure patients who initially presented with a weak retention after the hyperactive areas are destroyed. Regarding external beam radiation therapy, it should be considered that HCC is generally not a radio-resistant tumor but is located in an extremely radiosensitive organ. Accordingly, the dose delivered should not exceed 30 Gy on the whole liver as this is the threshold for radiation-induced liver disease. This dose level is less than standard tumor-killing doses for most of the other radiosensitive neoplasms. Advances in the field of radiotherapy planning have given the opportunity of dose escalation.

\section{Dosimetry and Safety on Workplace}

The rationale of radiotherapy includes the fact that the dose to be delivered for a therapeutic effect should be computed taking into account the dose delivered to critical organs. In case of ${ }^{131}$ I Lipiodol the accepted killing dose for HCC is $120 \mathrm{~Gy}$, being normal liver and lungs the critical organs.

For external beam radiotherapy it depends on fractionation and the total irradiation of liver and lungs should not exceed 30 Gy and 25 Gy, respectively [74], whereas the dose to critical organs is not exactly computable in case of metabolic radiotherapy. However, when ${ }^{90} \mathrm{Y}$ labeled microspheres are used the mean adsorbed dose in the liver is 120 Gy and more than $30 \mathrm{~Gy}$ in lungs. Apart from the radiometric issues related to the use of radioiodine (see body and hands exposure), it should be pointed out that the use of ${ }^{90} \mathrm{Y}$ may cause consistent exposure to the fingers of the personnel involved in the preparation and administration of the radiopharmaceuticals.

\section{Clinical Trials}

Considering ${ }^{131}$ I Lipiodol, a partial or incomplete response (reduction of tumor size and/or of hematic markers) was found in almost $71 \%$ of patients $[75,76]$. These results were confirmed also after correcting for the number of patients per study [77]. A complete response has been also described depending on type and HCC size. Multinodular forms showed a lower rate of response as well as those infiltrating. It has been demonstrated that the response rate is inversely correlated to the size of HCC, being $80 \%$ for nodules of less than $5 \mathrm{~cm}$ diameter [78] up to $22 \%$ for those of $10 \mathrm{~cm}$. However, the sole dimensional assessment of therapeutic response is not accurate since metabolic change should be taken into account. Accordingly, recent studies have shown the usefulness of positron emission tomography/computed tomography (PET/CT) for assessing the response to locoregional treatment of HCC [79] as demonstrated for other tumor types [80] and considering the potentialities of PET/CT methodology [81]. Moreover, other studies reported responses in $17 \%-92 \%$ of patients after ${ }^{131}$ I Lipiodol intra-arterial administration [77, 82]. LintiaGaultier et al. compared fifty patients with advanced HCC receiving intra-arterial injection of ${ }^{131} \mathrm{I}$ Lipiodol with 36 untreated patients. The ${ }^{131}$ I Lipiodol was associated with a survival benefit ( $32 \mathrm{wk}$ versus $8 \mathrm{wk}$ for the untreated group) and the 1-year survival rate was $32 \%$ versus $8 \%$ for the untreated group [19]. The transarterial metabolic radiotherapy has also been used for reducing the pain related to HCC whereas, in general and according to different trials, survival rates of 6 months are reported between 33 and $66 \%$ [83]. ${ }^{90} \mathrm{Y}$ microspheres can be used for treatment of large tumors of patients eligible for transplantation, in patients with portal vein thrombosis and with palliative intent [84]. The radioembolization of unresectable HCC with ${ }^{90} \mathrm{Y}$ microspheres is associated with acceptable toxicity and a favourable median survival time [85]. The intra-arterial administration of ${ }^{90} \mathrm{Y}$ microspheres has shown safety and effectiveness in both intermediate-stage and advanced-stage HCC [86]. This trend has been recently reported in retrospective and prospective studies [87-90]. The efficacy of external radiation therapy has been demonstrated in patients with small-size HCC noneligible for curative therapies [91]. Data from Mornex et al. (phase II study) confirmed the efficacy of radiation therapy in cirrhotic patients with one nodule $\leq 5 \mathrm{~cm}$ or two nodules $\leq 3 \mathrm{~cm}$. Tumor response was observed for $92 \%$ overall with $80 \%$ of complete response. After a mean follow-up of $29 \pm 9$ months, the recurrence rate was $22 \%$ and $41 \%$ for lesions inside and outside the irradiated volume, respectively [92].

Multicenter studies sponsored by the International Atomic Energy Agency (IAEA) showed that transarterial

${ }^{188}$ Re Lipiodol is a safe and cost-effective method to treat primary hepatocellular carcinoma. Immediate and late side effects were minimal. The objective response rate was $25 \%$ 
TABLE 2: Therapeutic strategies for nonoperable HCC.

\begin{tabular}{|c|c|c|c|c|}
\hline Treatment & Indications & Advantages & Disadvantages & Efficacy \\
\hline TACE & $\begin{array}{l}\text { (i) Large or multifocal HCC } \\
\text { not eligible for radical therapy } \\
\text { (ii) Nonoperable locoregional } \\
\text { disease without distant } \\
\text { metastases }\end{array}$ & $\begin{array}{l}\text { (i) Safety } \\
\text { (ii) Bridge modality in } \\
\text { patients awaiting OLT }\end{array}$ & $\begin{array}{l}\text { (i) Poor complete tumor response } \\
\text { (ii) Short time to progression } \\
\text { (iii) Short progression free survival } \\
\text { (iv) PES } \\
\text { (v) Ischemic damage to normal liver }\end{array}$ & $\begin{array}{l}\text { (i) Tumor shrinking } \\
\text { (ii) Survival (moderate) }\end{array}$ \\
\hline TAMR & $\begin{array}{l}\text { (i) Curative therapy of } \\
\text { small-size HCC } \\
\text { (ii) HCC with portal vein } \\
\text { thrombosis } \\
\text { (iii) Adjuvant treatment near } \\
\text { surgery } \\
\text { (iv) Neoadjuvant approach } \\
\text { (v) Palliation of nonoperable } \\
\text { HCC } \\
\text { (vi) Antalgic treatment for } \\
\text { hyperalgesic HCC }\end{array}$ & $\begin{array}{l}\text { (i) Cell killing intent } \\
\text { (ii) Healthy liver } \\
\text { preservation } \\
\text { (iii) Bridge modality in } \\
\text { patients awaiting OLT }\end{array}$ & $\begin{array}{l}\text { (i) Radiation pneumoni- } \\
\text { tis/hepatitis/cholecystitis/pancreatitis } \\
\text { (ii) Asthenia, anorexia, transient } \\
\text { hepatalgies, brief alterations of } \\
\text { hepatic tests, and leucopoenia } \\
\text { (iii) Workplace safety }\end{array}$ & $\begin{array}{l}\text { (i) Tumor shrinking } \\
\text { (ii) Survival benefit } \\
\text { (iii) Long term efficacy }\end{array}$ \\
\hline $\mathrm{RTx}^{*}$ & $\begin{array}{l}\text { (i) Nonoperable HCC } \\
\text { (ii) Unfeasible/failed } \\
\text { percutaneous therapies } \\
\text { (iii) Longest tumor diameter } \\
\leq 5.0 \mathrm{~cm} \\
\text { (iv) Child-Turcotte-Pugh } \\
\text { Class A or B } \\
\text { (v) Patients with 1-3 lesions } \\
\text { and sufficient uninvolved liver }\end{array}$ & $\begin{array}{l}\text { (i) Bridge modality in } \\
\text { patients awaiting OLT } \\
\text { (ii) Dose escalation } \\
\text { (iii) Accurate definition } \\
\text { of the target volume }\end{array}$ & (i) Surrounding healthy liver damage & $\begin{array}{l}\text { (i) Significant tumor } \\
\text { response in small-size } \\
\text { HCC }\end{array}$ \\
\hline
\end{tabular}

TACE: transarterial chemoembolization. OLT: orthotopic liver transplantation. PES: postembolization syndrome. TAMR: transarterial metabolic radiotherapy. RTx: external radiotherapy. *: including $3 \mathrm{D}$ conformal radiation techniques and stereotactic body radiotherapy.

and 1 - and 2 -year survivals were $46 \%$ and $23 \%$, respectively $[39,40]$.

\section{Still Open Questions and Combination of Therapies for HCC}

The efficacy of ${ }^{131}$ I Lipiodol has been evaluated versus chemoembolization. Patients complying with inoperable and nontransplantable HCC randomly underwent ${ }^{131}$ I Lipiodol or chemoembolization evaluating tumor size, alphafetoprotein levels, and survival rate. There was no significant difference in survival rate at 6 months and at four years between the two treatments as well as in terms of complete response. Both therapeutic methods showed similar effectiveness, although the ${ }^{131}$ I Lipiodol group exhibited better tolerance to treatment [93]. ${ }^{131}$ I Lipiodol was also shown to improve significantly the survival when compared to supportive medical therapy [63]. It seems clear that TAMR with ${ }^{131} \mathrm{I}$ Lipiodol is at least as effective as the TACE, showing a better safety profile. This treatment has been also used with adjuvant intent and patients compared to a control group. The survival rate at 3 years in the treatment group and controls was $86.4 \%$ and $43.6 \%$, respectively [62]. ${ }^{131}$ I Lipiodol has also been combined with low-dose of cisplatin and patients who received both treatments showed a response rate of $90 \%$, as compared to those who received ${ }^{131} \mathrm{I}$ Lipiodol alone [94]. This combined approach is important and a synergy between radiotherapy and chemotherapy can be envisaged. This approach was already tested using ${ }^{90} \mathrm{Y}$ microspheres in patients with hepatic metastases [95]. Actually, there are no published studies endorsing the combination of ${ }^{131} \mathrm{I}$ Lipiodol with external radiotherapy, but indeed it represents an intriguing area of research similar to that combining hyperthermia with transarterial metabolic radiotherapy. Recently, the efficacy of ${ }^{90} \mathrm{Y}$ microspheres has been compared with transarterial chemoembolization for treating HCC [96]. No significant differences were found in progression free survival, overall survival, and time to progression. The lower rate of tumor progression in radiotreated group was nullified by a greater incidence of liver failure [96]. Both these modalities can be alternatively used in case of unresectable HCC, as first-line treatment [97]. Concerning the combination of ${ }^{90} \mathrm{Y}$ microspheres with other treatment modalities, a plethora of studies has recently shown that radioembolization followed by an inhibitor of tyrosine protein kinases (sorafenib) appears to be as well-tolerated as the inhibitor alone [98-100]. Conformal radiotherapy combined with hepatic arterial floxuridine has improved the survival of patients with intrahepatic cancer ineligible for surgical resection or ablation as reported by Ben-Josef et al. [98]. Moreover, the total radiation dose was the only significant factor for survival. As for other cancer types and other aggregate therapies, a cumulative tumor-killing effect can be postulated when using a combination of RTx and inhibitor of tyrosine protein kinases $[101,102]$. This captivating area of research (Table 2) demands further preclinical and clinical 
TABLE 3: Summary of recent clinical trials considering radiation-based therapies (internal and external) for HCC.

\begin{tabular}{|c|c|c|c|}
\hline Reference & Year & Therapy & Findings \\
\hline$[75]$ & 1988 & ${ }^{131}$ I Lipiodol & $\begin{array}{l}50 \% \text { tumor size reduction, response rate } \\
60 \%\end{array}$ \\
\hline$[77]$ & 1991 & ${ }^{131}$ I Lipiodol & $\begin{array}{l}\text { Response rate } 88.9 \%-25 \% \text { according to } \\
\text { tumor size }\end{array}$ \\
\hline [83] & 1992 & ${ }^{131}$ I Lipiodol & Decrease of pain in $33 \%-66 \%$ \\
\hline [93] & 1997 & ${ }^{131}$ I Lipiodol versus TACE & ${ }^{131}$ I Lipiodol and TACE equally effective \\
\hline [95] & 2001 & $\begin{array}{l}{ }^{90} \mathrm{Y} \text { microspheres plus } \mathrm{CHx} \\
\text { versus } \mathrm{CHx} \text { alone }\end{array}$ & $\begin{array}{l}\text { Tumor response } 44 \% \text { combined therapy } \\
\text { versus } 17.6 \% \mathrm{CHx} \text { alone }\end{array}$ \\
\hline$[94]$ & 2002 & ${ }^{131}$ I Lipiodol plus cisplatin & $\begin{array}{l}\text { Response rate } 90 \% \text { combined therapy } \\
\text { versus } 40 \%{ }^{131} \text { I Lipiodol alone }\end{array}$ \\
\hline [91] & 2005 & $\mathrm{RTx}$ & Tumor response $66.1 \%$ \\
\hline [98] & 2005 & RTx plus floxuridine & $\begin{array}{l}\text { Improved survival in patients with } \\
\text { unresectable intrahepatic malignancies }\end{array}$ \\
\hline [82] & 2005 & ${ }^{131}$ I Lipiodol & Response rate $17 \%-92 \%$ \\
\hline [87] & 2006 & ${ }^{90} \mathrm{Y}$ microspheres & $\begin{array}{l}\text { Disease control rate } 100 \% \text { and response } \\
\text { rate } 23.8 \%\end{array}$ \\
\hline$[92]$ & 2006 & $\mathrm{RTx}$ & $\begin{array}{l}\text { Response rate } 92 \% \text { in patients with } \\
\text { small-size HCC }\end{array}$ \\
\hline [39] & 2008 & ${ }^{188}$ Re Lipiodol & $\begin{array}{l}\text { objective response rate } 25 \% \\
2 \text {-year survival } 23 \%\end{array}$ \\
\hline [85] & 2012 & ${ }^{90} \mathrm{Y}$ microspheres & Favourable median survival time \\
\hline [19] & 2013 & ${ }^{131}$ I Lipiodol & $\begin{array}{l}\text { Survival benefit; } 32 \% \text { of treated versus } 8 \% \\
\text { of untreated pts }\end{array}$ \\
\hline [99] & 2014 & ${ }^{90} \mathrm{Y}$ microspheres plus sorafenib & Potential efficacy and manageable toxicity \\
\hline$[86]$ & 2015 & ${ }^{90} \mathrm{Y}$ microspheres & $\begin{array}{l}\text { Safe and effective in both intermediate- } \\
\text { and advanced stage }\end{array}$ \\
\hline [96] & 2015 & ${ }^{90} \mathrm{Y}$ microspheres versus TACE & $\begin{array}{l}\text { No significant differences in PFS, OS, and } \\
\text { TTP }\end{array}$ \\
\hline$[100]$ & 2015 & ${ }^{90} \mathrm{Y}$ microspheres plus sorafenib & $\begin{array}{l}{ }^{90} \mathrm{Y} \text { microspheres plus sorafenib } \\
\text { well-tolerated as sorafenib alone }\end{array}$ \\
\hline [88] & 2016 & $\begin{array}{l}{ }^{90} \mathrm{Y} \text { microspheres versus } \\
\text { sorafenib }\end{array}$ & $\begin{array}{l}{ }^{90} \mathrm{Y} \text { microspheres more effective than } \\
\text { sorafenib in patients with PVT }\end{array}$ \\
\hline
\end{tabular}

Radionuclides administration by transarterial approach. TACE: transarterial chemoembolization. CHx: chemotherapy. PFS: progression free survival. OS: overall survival. TTP: time to progression. PVT: portal vein thrombosis. RTx: external radiotherapy including conformal radiation techniques.

trials in order to offer new therapeutic options for HCC (Table 3).

\section{Economics}

The cost of the commercially available radiopharmaceuticals ${ }^{131} \mathrm{I}$ Lipiodol and ${ }^{90} \mathrm{Y}$ microsphere is prohibitively high for developing countries where the HCC represents an impending social problem. The actual economic context requires that a treatment should be cost-effective, easy to transport, and uncomplicated to administer and should not create radiation protection issues. All these topics contribute to the global cost of transarterial metabolic radiotherapy for hepatocellular carcinoma, each of them with a different but definite weight. The current price of ${ }^{188}$ Re generator can be considered convenient for developed countries, but it will constrain wide clinical application of ${ }^{188} \mathrm{Re}$ in developing countries. In general, the accurate assessment of these costs is complicated. However, it should be pointed out that radiation-based therapy for HCC determines less side effects as compared to other modalities reducing the costs for supportive therapies and warranting adequate quality of life.

\section{Conclusion}

The role of transarterial metabolic radiotherapy for hepatocellular carcinoma has gained full consideration over the years. ${ }^{131} \mathrm{I}$ Lipiodol and ${ }^{90} \mathrm{Y}$ microspheres have proven effectiveness in the treatment of inoperable HCC also with portal vein thrombosis. This therapy can also be implemented with either neoadjuvant or adjuvant intent, reducing the risk of recurrence. It has been recognized as effective as chemoembolization. The transarterial metabolic radiotherapy is more effective than palliative supportive therapies and can be used for dealing with pain in the event of hyperalgesic HCC. Contraindications are clearly defined and 
side effects are controllable and predictable, supporting this treatment as being well-tolerated. Radiation protection and manipulation issues are manageable. Transarterial metabolic radiotherapy alone or in combination with other local or systemic therapies offers encouraging results on tumor local control and survival. The literature also supports the efficacy and safety of external radiation therapy for HCC that has until now been considered a radio-resistant tumor.

\section{Additional Points}

Core tips: treatment of hepatocellular carcinoma is challenging since it is frequently associated with a causal liver morbidity. The role of radiation-based locoregional therapy has progressed regardless of few side effects. Radiopharmaceutical and technical advances hold great promise for patients who are not eligible for radical treatments. The combination of different therapeutic approaches constitutes an interesting option as well.

\section{Competing Interests}

The authors have indicated they have no financial competing interests.

\section{References}

[1] R. Dhanasekaran, A. Limaye, and R. Cabrera, "Hepatocellular carcinoma: current trends in worldwide epidemiology, risk factors, diagnosis, and therapeutics," Hepatic Medicine: Evidence and Research, vol. 4, pp. 19-37, 2012.

[2] Surveillance Research Program, National Cancer Institute Fast stats: an interactive tool for access to SEER cancer statistics, November 2011, http://seer.cancer.gov/faststats.

[3] J. Ferlay, H.-R. Shin, F. Bray, D. Forman, C. Mathers, and D. M. Parkin, "Estimates of worldwide burden of cancer in 2008: GLOBOCAN 2008," International Journal of Cancer, vol. 127, no. 12, pp. 2893-2917, 2010.

[4] K. Okuda, "Hepatocellular carcinoma," Journal of Hepatology, vol. 32, no. 1, supplement, pp. 225-237, 2000.

[5] H. B. El-Serag and A. C. Mason, "Rising incidence of hepatocellular carcinoma in the United States," The New England Journal of Medicine, vol. 340, no. 10, pp. 745-750, 1999.

[6] S. Deuffic, L. Buffat, T. Poynard, and A. J. Valleron, "Modeling the hepatitis C virus epidemic in France," Hepatology, vol. 29, no. 5, pp. 1596-1601, 1999.

[7] M. C. Wallace, D. Preen, G. P. Jeffrey, and L. A. Adams, "The evolving epidemiology of hepatocellular carcinoma: a global perspective," Expert Review of Gastroenterology and Hepatology, vol. 9, no. 6, pp. 765-779, 2015.

[8] T. P. Hong, P. Gow, M. Fink et al., "Novel population-based study finding higher than reported hepatocellular carcinoma incidence suggests an updated approach is needed," Hepatology, vol. 63, no. 4, pp. 1205-1212, 2016.

[9] K. Joshi, M. Mendler, R. Gish et al., "Hepatocellular carcinoma surveillance: a national survey of current practices in the USA," Digestive Diseases and Sciences, vol. 59, no. 12, pp. 3073-3077, 2014.

[10] R. Lencioni, "Chemoembolization in patients with hepatocellular carcinoma," Liver Cancer, vol. 1, no. 1, pp. 41-50, 2012.
[11] E. Liapi and J.-F. Geschwind, "Interventional oncology: new options for interstitial treatments and intravascular approaches: targeting tumor metabolism via a loco-regional approach: a new therapy against liver cancer," Journal of Hepato-BiliaryPancreatic Sciences, vol. 17, no. 4, pp. 405-406, 2010.

[12] W. D. Hsiao, C. Y. Peng, P. H. Chuang et al., "Evaluation of doseefficacy of sorafenib and effect of transarterial chemoembolization in hepatocellular carcinoma patients: a retrospective study," BMC Gastroenterology, vol. 16, no. 1, p. 50, 2016.

[13] T. Yamasaki, S. Hamabe, I. Saeki et al., "A novel transcatheter arterial infusion chemotherapy using iodized oil and degradable starch microspheres for hepatocellular carcinoma: a prospective randomized trial," Journal of Gastroenterology, vol. 46, no. 3, pp. 359-366, 2011.

[14] L. Vadot, M. Boulin, B. Guiu et al., "Clinical and economic impact of drug eluting beads in transarterial chemoembolization for hepatocellular carcinoma," Journal of Clinical Pharmacy and Therapeutics, vol. 40, no. 1, pp. 83-90, 2015.

[15] S. C. H. Yu, J. W. Y. Hui, E. P. Hui et al., "Unresectable hepatocellular carcinoma: randomized controlled trial of transarterial ethanol ablation versus transcatheter arterial chemoembolization," Radiology, vol. 270, no. 2, pp. 607-620, 2014.

[16] G. F. Stefanini, P. Amorati, M. Biselli et al., "Efficacy of transarterial targeted treatments on survival of patients with hepatocellular carcinoma: an Italian experience," Cancer, vol. 75, no. 10, pp. 2427-2434, 1995.

[17] G. Pelletier, A. Roche, O. Ink et al., "A randomized trial of hepatic arterial chemoembolization in patients with unresectable hepatocellular carcinoma," Journal of Hepatology, vol. 11, no. 2, pp. 181-184, 1990.

[18] J. W. Lee, J. K. Oh, Y. A. Chung et al., "Prognostic significance of ${ }^{18}$ F-FDG uptake in hepatocellular carcinoma treated with transarterial chemoembolization or concurrent chemoradiotherapy: a multicenter retrospective cohort study," Journal of Nuclear Medicine, vol. 57, no. 4, pp. 509-516, 2016.

[19] A. Lintia-Gaultier, C. Perret, C. Ansquer, T. Eugène, F. KraeberBodéré, and E. Frampas, "Intra-arterial injection of 131I-labeled Lipiodol for advanced hepatocellular carcinoma: a 7 years' experience," Nuclear Medicine Communications, vol. 34, no. 7, pp. 674-681, 2013.

[20] S. Subramanian, T. Das, S. Chakraborty et al., "Preparation of 177Lu-labeled oxine in lipiodol as a possible agent for therapy of hepatocellular carcinoma: A Preliminary Animal Study," Cancer Biotherapy and Radiopharmaceuticals, vol. 25, no. 5, pp. 539543, 2010.

[21] B. Lambert, K. Bacher, and L. Defreyne, "Rhenium-188 based radiopharmaceuticals for treatment of liver tumours," Quarterly Journal of Nuclear Medicine and Molecular Imaging, vol. 53, no. 3, pp. 305-310, 2009.

[22] T. Das, S. Chakraborty, H. D. Sarma, M. Venkatesh, and S. Banerjee, "Preparation of 166Ho-oxine-lipiodol and its preliminary bioevaluation for the potential application in therapy of liver cancer," Nuclear Medicine Communications, vol. 30, no. 5, pp. 362-367, 2009.

[23] J. Yu, U. O. Häfeli, M. Sands, and Y. Dong, "90Y-oxineethiodol, a potential radiopharmaceutical for the treatment of liver cancer," Applied Radiation and Isotopes, vol. 58, no. 5, pp. 567-573, 2003.

[24] S. Ho, J. W. Y. Lau, T. W. T. Leung, J. E. Dancey, and J. Goin, "Intrahepatic 90Y-microspheres for hepatocellular carcinoma," Journal of Nuclear Medicine, vol. 42, no. 10, pp. 1587-1589, 2001. 
[25] Z.-C. Zeng, Z.-Y. Tang, K.-D. Liu, J.-Z. Lu, H. Xie, and Z. Yao, "Improved long-term survival for unresectable hepatocellular carcinoma (HCC) with a combination of surgery and intrahepatic arterial infusion of 131I-anti-HCC mAb. Phase I/II clinical trials," Journal of Cancer Research and Clinical Oncology, vol. 124, no. 5, pp. 275-280, 1998.

[26] J. Liebster and V. Kočandrle, "Exchange labelling of 'lipiodol' ultra fluids with lodine-131 and its intra-lymphatic administration with a fistula on the thoracic duct," Nature, vol. 203, no. 4946, pp. 777-778, 1964.

[27] J. L. Raoul, R. Duvauferrier, P. Bourguet et al., "Lipiodolized angiography in hepatocellular carcinomas. Contribution of iodine-131-labelled Lipiodol," Journal de Radiologie, vol. 67, no. 11, pp. 797-801, 1986.

[28] D. M. Biederman, J. J. Titano, K. M. Lee et al., "Yttrium-90 glassbased microsphere radioembolization in the treatment of hepatocellular carcinoma secondary to the hepatitis B virus: safety, efficacy, and survival," Journal of Vascular and Interventional Radiology, vol. 26, no. 11, pp. 1630-1638, 2015.

[29] N. Khajornjiraphan, N. A. Thu, and P. K. Chow, "Yttrium-90 microspheres: a review of its emerging clinical indications," Liver Cancer, vol. 4, no. 1, pp. 6-15, 2015.

[30] M. Xi, M.-Z. Liu, X.-W. Deng et al., "Defining internal target volume (ITV) for hepatocellular carcinoma using fourdimensional CT," Radiotherapy and Oncology, vol. 84, no. 3, pp. 272-278, 2007.

[31] R. P. Beasley, C.-C. Lin, L.-Y. Hwang, and C.-S. Chien, "Hepatocellular carcinoma and hepatitis B virus. A prospective study of 22707 men in Taiwan," The Lancet, vol. 318, no. 8256, pp. 1129$1133,1981$.

[32] I. H. McKillop, D. M. Moran, X. Jin, and L. G. Koniaris, "Molecular pathogenesis of hepatocellular carcinoma," Journal of Surgical Research, vol. 136, no. 1, pp. 125-135, 2006.

[33] C. Guha and B. D. Kavanagh, "Hepatic radiation toxicity: avoidance and amelioration," Seminars in Radiation Oncology, vol. 21, no. 4, pp. 256-263, 2011.

[34] C. H. Park, J. H. Suh, H. S. Yoo, J. T. Lee, and D. I. Kim, "Evaluation of intrahepatic I-131 ethiodol on a patient with hepatocellular carcinoma therapeutic feasibility study," Clinical Nuclear Medicine, vol. 11, no. 7, pp. 514-517, 1986.

[35] F. I. Chou, K. C. Fang, C. Chung et al., "Lipiodol uptake and retention by human hepatoma cells," Nuclear Medicine and Biology, vol. 22, no. 3, pp. 379-386, 1995.

[36] C. Park, S. I. Choi, H. Kim, H. S. Yoo, and Y. B. Lee, "Distribution of Lipiodol in hepatocellular carcinoma," Liver, vol. 10, no. 2, pp. 72-78, 1990.

[37] R. E. Hind, M. Loizidou, S. Perring et al., "Biodistribution of Lipiodol following hepatic arterial injection," British Journal of Surgery, vol. 79, no. 9, pp. 952-954, 1992.

[38] M. Nakajo, H. Kobayashi, K. Shimabukuro et al., "Biodistribution and in vivo kinetics of iodine-131 lipiodol infused via the hepatic artery of patients with hepatic cancer," Journal of Nuclear Medicine, vol. 29, no. 6, pp. 1066-1077, 1988.

[39] P. Bernal, J.-L. Raoul, J. Stare et al., "International atomic energy agency-sponsored multination study of intra-arterial rhenium-188-labeled lipiodol in the treatment of inoperable hepatocellular carcinoma: results with special emphasis on prognostic value of dosimetric study," Seminars in Nuclear Medicine, vol. 38, no. 2, pp. S40-S45, 2008.

[40] P. Bernal, J.-L. Raoul, G. Vidmar et al., "Intra-arterial rhenium188 lipiodol in the treatment of inoperable hepatocellular carcinoma: results of an IAEA-Sponsored Multination Study," International Journal of Radiation Oncology Biology Physics, vol. 69, no. 5, pp. 1448-1455, 2007.

[41] J. M. Jeong, Y. J. Kim, Y. S. Lee et al., "Lipiodol solution of a lipophilic agent, ${ }^{188} \mathrm{Re}-\mathrm{TDD}$, for the treatment of liver cancer," Nuclear Medicine and Biology, vol. 28, no. 2, pp. 197-204, 2001.

[42] S.-J. Wang, W.-Y. Lin, W.-Y. Lui, M.-N. Chen, Z.-T. Tsai, and G. Ting, "Hepatic artery injection of yttrium-90-lipiodol: biodistribution in rats with hepatoma," Journal of Nuclear Medicine, vol. 37, no. 2, pp. 332-335, 1996.

[43] V. Vilgrain, M. Abdel-Rehim, A. Sibert et al., "Radioembolisation with yttrium-90 microspheres versus sorafenib for treatment of advanced hepatocellular carcinoma (SARAH): study protocol for a randomised controlled trial," Trials, vol. 15, article 474, 2014.

[44] S. A. Gulec, G. Mesoloras, W. A. Dezarn, P. McNeillie, and A. S. Kennedy, "Safety and efficacy of Y-90 microsphere treatment in patients with primary and metastatic liver cancer: The tumor selectivity of the treatment as a function of tumor to liver flow ratio," Journal of Translational Medicine, vol. 5, article 15, 2007.

[45] W.-Y. Lau, W.-T. Leung, S. Ho et al., "Treatment of inoperable hepatocellular carcinoma with intrahepatic arterial yttrium-90 microspheres: a phase I and II study," British Journal of Cancer, vol. 70, no. 5, pp. 994-999, 1994.

[46] A. Nardelli, E. Castaldi, G. Ortosecco et al., "Enhancement of reaction conditions for the radiolabelling of DOTA-peptides with high activities of yttrium-90," Applied Radiation and Isotopes, vol. 69, no. 1, pp. 52-55, 2011.

[47] G. Storto, A. De Renzo, T. Pellegrino et al., "Assessment of metabolic response to radioimmunotherapy with 90Yibritumomab tiuxetan in patients with relapsed or refractory B-cell non-Hodgkin lymphoma," Radiology, vol. 254, no. 1, pp. 245-252, 2010.

[48] H. Sharma, "Role of external beam radiation therapy in management of hepatocellular carcinoma," Journal of Clinical and Experimental Hepatology, vol. 4, supplement 3, pp. S122-S125, 2014.

[49] Y. Tanaka, T. Nakazawa, S. Komori et al., "Radiotherapy for patients with unresectable advanced hepatocellular carcinoma with invasion to intrahepatic large vessels: efficacy and outcomes," Journal of Gastroenterology and Hepatology, vol. 29, no. 2, pp. 352-357, 2014.

[50] T. S. Lawrence, M. L. Kessler, and J. M. Robertson, "Conformal high-dose radiation plus intraarterial floxuridine for hepatic cancer," Oncology, vol. 7, no. 10, pp. 51-57, 1993.

[51] J. M. Robertson, T. S. Lawrence, J. C. Andrews, S. Walker, M. L. Kessler, and W. D. Ensminger, "Long-term results of hepatic artery fluorodeoxyuridine and conformal radiation therapy for primary hepatobiliary cancers," International Journal of Radiation Oncology, Biology, Physics, vol. 37, no. 2, pp. 325-330, 1997.

[52] S. H. Bae, M.-S. Kim, C. K. Cho, K. B. Kim, C. J. Han, and Y. H. Kim, "Feasibility and efficacy of stereotactic ablative radiotherapy for: Barcelona clinic liver cancer-C stage hepatocellular carcinoma," Journal of Korean Medical Science, vol. 28, no. 2, pp. 213-219, 2013.

[53] A. Bujold, C. A. Massey, J. J. Kim et al., "Sequential phase I and II trials of stereotactic body radiotherapy for locally advanced hepatocellular carcinoma," Journal of Clinical Oncology, vol. 31, no. 13, pp. 1631-1639, 2013. 
[54] W.-Y. Huang, Y.-M. Jen, M.-S. Lee et al., "Stereotactic body radiation therapy in recurrent hepatocellular carcinoma," International Journal of Radiation Oncology, Biology, Physics, vol. 84, no. 2, pp. 355-361, 2012.

[55] H. R. Cárdenes, T. R. Price, S. M. Perkins et al., "Phase I feasibility trial of stereotactic body radiation therapy for primary hepatocellular carcinoma," Clinical and Translational Oncology, vol. 12, no. 3, pp. 218-225, 2010.

[56] J. H. Kwon, S. H. Bae, J. Y. Kim et al., "Long-term effect of stereotactic body radiation therapy for primary hepatocellular carcinoma ineligible for local ablation therapy or surgical resection. Stereotactic radiotherapy for liver cancer," BMC Cancer, vol. 10, article 475, 2010.

[57] Y. S. Seo, M.-S. Kim, S. Y. Yoo et al., "Preliminary result of stereotactic body radiotherapy as a local salvage treatment for inoperable hepatocellular carcinoma," Journal of Surgical Oncology, vol. 102, no. 3, pp. 209-214, 2010.

[58] A. W. Katz, S. Chawla, Z. Qu, R. Kashyap, M. T. Milano, and A. F. Hezel, "Stereotactic hypofractionated radiation therapy as a bridge to transplantation for hepatocellular carcinoma: clinical outcome and pathologic correlation," International Journal of Radiation Oncology Biology Physics, vol. 83, no. 3, pp. 895-900, 2012.

[59] J. K. O’Connor, J. Trotter, G. L. Davis, J. Dempster, G. B. Klintmalm, and R. M. Goldstein, "Long-term outcomes of stereotactic body radiation therapy in the treatment of hepatocellular cancer as a bridge to transplantation," Liver Transplantation, vol. 18, no. 8, pp. 949-954, 2012.

[60] A. Al-Kalbani and Y. Kamel, "Y-90 microshperes in the treatment of unresectable hepatocellular carcinoma," Saudi Journal of Gastroenterology, vol. 14, no. 2, pp. 90-92, 2008.

[61] J.-L. Raoul, D. Guyader, J.-F. Bretagne et al., "Randomized controlled trial for hepatocellular carcinoma with portal vein thrombosis: intra-arterial iodine-131-iodized oil versus medical support," Journal of Nuclear Medicine, vol. 35, no. 11, pp. 17821787, 1994.

[62] W. Y. Lau, T. W. T. Leung, S. K. W. Ho et al., "Adjuvant intraarterial iodine-131-labelled lipiodol for resectable hepatocellular carcinoma: a prospective randomised trial," The Lancet, vol. 353, no. 9155 , pp. 797-801, 1999.

[63] R. T.-P. Poon, S.-T. Fan, I. O.-L. Ng, C.-M. Lo, C.-L. Liu, and J. Wong, "Different risk factors and prognosis for early and late intrahepatic recurrence after resection of hepatocellular carcinoma," Cancer, vol. 89, no. 3, pp. 500-507, 2000.

[64] T. S. Su, P. Liang, H. Z. Lu et al., "Stereotactic body radiation therapy for small primary or recurrent hepatocellular carcinoma in 132 Chinese patients," Journal of Surgical Oncology, vol. 113, no. 2, pp. 181-187, 2016.

[65] T. Kimura, H. Aikata, S. Takahashi et al., "Stereotactic body radiotherapy for patients with small hepatocellular carcinoma ineligible for resection or ablation therapies," Hepatology Research, vol. 45, no. 4, pp. 378-386, 2015.

[66] N. Sanuki, A. Takeda, Y. Oku et al., "Stereotactic body radiotherapy for small hepatocellular carcinoma: a retrospective outcome analysis in 185 patients," Acta Oncologica, vol. 53, no. 3, pp. 399404, 2014.

[67] European Association for the Study of the Liver and European Organisation for Research and Treatment of Cancer, "EASLEORTC clinical practice guidelines: management of hepatocellular carcinoma," Journal of Hepatology, vol. 56, no. 4, pp. 908943, 2012.
[68] C. L. Maini, M. G. Scelsa, C. Fiumara et al., "Superselective intra-arterial radiometabolic therapy with I-131 lipiodol in hepatocellular carcinoma," Clinical Nuclear Medicine, vol. 21, no. 3, pp. 221-226, 1996.

[69] T. de Baere, P. Taourel, J. M. Tubiana et al., "Hepatic intraarterial 131I iodized oil for treatment of hepatocellular carcinoma in patients with impeded portal venous flow," Radiology, vol. 212, no. 3, pp. 665-668, 1999.

[70] C. H. Park, J. H. Suh, H. S. Yoo, J. T. Lee, D. I. Kim, and B. S. Kim, "Treatment of hepatocellular carcinoma (HCC), with radiolabeled lipiodol: A Preliminary Report," Nuclear Medicine Communications, vol. 8, no. 12, pp. 1075-1087, 1987.

[71] H. S. Yoo, C. H. Park, J. T. Lee et al., "Small hepatocellular carcinoma: high dose internal radiation therapy with superselective intra-arterial injection of I-131-labeled Lipiodol," Cancer Chemotherapy and Pharmacology, vol. 33, no. 1, pp. S128-S133, 1994.

[72] Y. Yumoto, K. Jinno, S. Inatsuki et al., "Treatment of hepatocellular carcinoma by transcatheter hepatic arterial injection of radioactive iodized oil solution," Cancer Chemotherapy and Pharmacology, vol. 31, no. 1, pp. S128-S136, 1992.

[73] N. Heger, S. Bayindir, R. Steckenmesser, and H. Schirmer, "Percutaneous catheter-arteriographies: seldinger technique," Minnesota Medicine, vol. 53, no. 10, pp. 1093-1097, 1970.

[74] N. Iwata, Y. Seto, and E. Kakishita, "Radiation hepatitis," Ryōikibetsu Shōkōgun Shirìzu, vol. 8, pp. 588-590, 1995.

[75] J.-F. Bretagne, J.-L. Raoul, P. Bourguet et al., "Hepatic artery injection of I-131-labeled Lipiodol. Part II. Preliminary results of therapeutic use in patients with hepatocellular carcinoma and liver metastases," Radiology, vol. 168, no. 2, pp. 547-550, 1988.

[76] Y. Kondo, O. Kimura, and T. Shimosegawa, "Radiation therapy has been shown to be adaptable for various stages of hepatocellular carcinoma," World Journal of Gastroenterology, vol. 21, no. 1, pp. 94-101, 2015.

[77] H. S. Yoo, J. T. Lee, K. W. Kim et al., "Nodular hepatocellular carcinoma. Treatment with subsegmental intraarterial injection of iodine 131-labeled iodized oil," Cancer, vol. 68, no. 9, pp. 1878$1884,1991$.

[78] H. S. Yoo, C. H. Park, J. H. Suh et al., "Radioiodinated fatty acid esters in the management of hepatocellular carcinoma: preliminary findings," Cancer Chemotherapy and Pharmacology, vol. 23, no. 1, pp. S54-S58, 1989.

[79] T. Torizuka, N. Tamaki, T. Inokuma et al., "Value of fluorine-18FDG-PET to monitor hepatocellular carcinoma after interventional therapy," Journal of Nuclear Medicine, vol. 35, no. 12, pp. 1965-1969, 1994.

[80] G. Storto, E. Nicolai, and M. Salvatore, "[18F]FDG-PET-CT for early monitoring of tumor response: when and why," Quarterly Journal of Nuclear Medicine and Molecular Imaging, vol. 53, no. 2, pp. 167-180, 2009.

[81] F. Maddalena, G. Lettini, R. Gallicchio et al., "Evaluation of glucose uptake in normal and cancer cell lines by positron emission tomography," Molecular Imaging, vol. 14, no. 8, pp. 490-498, 2015.

[82] B. Lambert and C. Van de Wiele, "Treatment of hepatocellular carcinoma by means of radiopharmaceuticals," European Journal of Nuclear Medicine and Molecular Imaging, vol. 32, no. 8, pp. 980-989, 2005.

[83] J. I. Raoul, J. F. Bretagne, J. P. Caucanas et al., "Internal radiation therapy for hepatocellular carcinoma: results of a French multicenter Phase II trial of transarterial injection of 
iodine 131- labeled Lipiodol," Cancer, vol. 69, no. 2, pp. 346-352, 1992.

[84] K. Memon, R. J. Lewandowski, A. Riaz, and R. Salem, "Yttrium 90 microspheres for the treatment of hepatocellular carcinoma," Recent Results in Cancer Research, vol. 190, pp. 207-224, 2013.

[85] S. M. Ibrahim, L. Kulik, T. Baker et al., "Treating and downstaging hepatocellular carcinoma in the caudate lobe with yttrium90 radioembolization," Cardio Vascular and Interventional Radiology, vol. 35, no. 5, pp. 1094-1101, 2012.

[86] Y. Kim do, B. J. Park, Y. H. Kim et al., "Radioembolization with yttrium-90 resin microspheres in hepatocellular carcinoma: a multicenter prospective study," American Journal of Clinical Oncology, vol. 38, no. 5, pp. 495-501, 2015.

[87] B. Sangro, J. I. Bilbao, J. Boan et al., "Radioembolization using 90Y-resin microspheres for patients with advanced hepatocellular carcinoma," International Journal of Radiation Oncology Biology Physics, vol. 66, no. 3, pp. 792-800, 2006.

[88] J. Edeline, L. Crouzet, B. Campillo-Gimenez et al., "Selective internal radiation therapy compared with sorafenib for hepatocellular carcinoma with portal vein thrombosis," European Journal of Nuclear Medicine and Molecular Imaging, vol. 43, no. 4, pp. 635-643, 2016.

[89] L. Kulik and R. Salem, "Downstaging: looking for answers, generating more questions?" Liver Transplantation, vol. 21, no. 9, pp. 1117-1119, 2015.

[90] W. Y. Lau, S. Ho, T. W. T. Leung et al., "Selective internal radiation therapy for nonresectable hepatocellular carcinoma with intraarterial infusion of 90yttrium microspheres," International Journal of Radiation Oncology Biology Physics, vol. 40, no. 3, pp. 583-592, 1998.

[91] W. Park, D. H. Lim, S. W. Paik et al., "Local radiotherapy for patients with unresectable hepatocellular carcinoma," International Journal of Radiation Oncology Biology Physics, vol. 61, no. 4, pp. 1143-1150, 2005.

[92] F. Mornex, N. Girard, C. Beziat et al., "Feasibility and efficacy of high-dose three-dimensional-conformal radiotherapy in cirrhotic patients with small-size hepatocellular carcinoma noneligible for curative therapies-mature results of the French Phase II RTF-1 trial," International Journal of Radiation Oncology, Biology, Physics, vol. 66, no. 4, pp. 1152-1158, 2006.

[93] J.-L. Raoul, D. Guyader, J.-F. Bretagne et al., "Prospective randomized trial of chemoembolization versus intraarterial injection of 131I-labeled-iodized oil in the treatment of hepatocellular carcinoma," Hepatology, vol. 26, no. 5, pp. 1156-1161, 1997.

[94] B. Brans, K. Van Laere, F. Gemmel et al., "Combining iodine131 Lipiodol therapy with low-dose cisplatin as a radiosensitiser: preliminary results in hepatocellular carcinoma," European Journal of Nuclear Medicine, vol. 29, no. 7, pp. 928-932, 2002.

[95] B. Gray, G. Van Hazel, M. Hope et al., "Randomised trial of SIRSpheres plus chemotherapy vs. chemotherapy alone for treating patients with liver metastases from primary large bowel cancer," Annals of Oncology, vol. 12, no. 12, pp. 1711-1720, 2001.

[96] M. B. Pitton, R. Kloeckner, C. Ruckes et al., "Randomized comparison of selective internal radiotherapy (SIRT) versus drugeluting bead transarterial chemoembolization (DEB-TACE) for the treatment of hepatocellular carcinoma," CardioVascular and Interventional Radiology, vol. 38, no. 2, pp. 352-360, 2015.

[97] F. T. Kolligs, J. I. Bilbao, T. Jakobs et al., "Pilot randomized trial of selective internal radiation therapy vs. chemoembolization in unresectable hepatocellular carcinoma," Liver International, vol. 35, no. 6, pp. 1715-1721, 2015.
[98] E. Ben-Josef, D. Normolle, W. D. Ensminger et al., "Phase II trial of high-dose conformal radiation therapy with concurrent hepatic artery floxuridine for unresectable intrahepatic malignancies," Journal of Clinical Oncology, vol. 23, no. 34, pp. 87398747, 2005.

[99] P. K. H. Chow, D. Y. H. Poon, M.-W. Khin et al., "Multicenter phase II study of sequential radioembolization-sorafenib therapy for inoperable hepatocellular carcinoma," PLoS ONE, vol. 9, no. 3, Article ID e90909, 2014.

[100] J. Ricke, K. Bulla, F. Kolligs et al., "Safety and toxicity of radioembolization plus Sorafenib in advanced hepatocellular carcinoma: analysis of the European multicentre trial SORAMIC," Liver International, vol. 35, no. 2, pp. 620-626, 2015.

[101] P. Pedicini, R. Caivano, B. A. Jereczek-Fossa et al., "Modelling the correlation between EGFr expression and tumour cell radiosensitivity, and combined treatments of radiation and monoclonal antibody EGFr inhibitors," Theoretical Biology and Medical Modelling, vol. 9, article 23, 2012.

[102] P. Pedicini, A. Nappi, L. Strigari et al., "Correlation between egfr expression and accelerated proliferation during radiotherapy of head and neck squamous cell carcinoma," Radiation Oncology, vol. 7, article 143, 2012. 


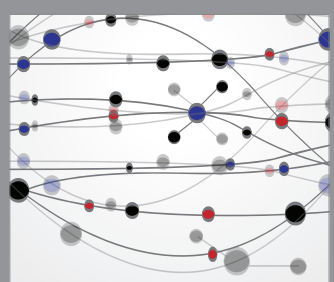

The Scientific World Journal
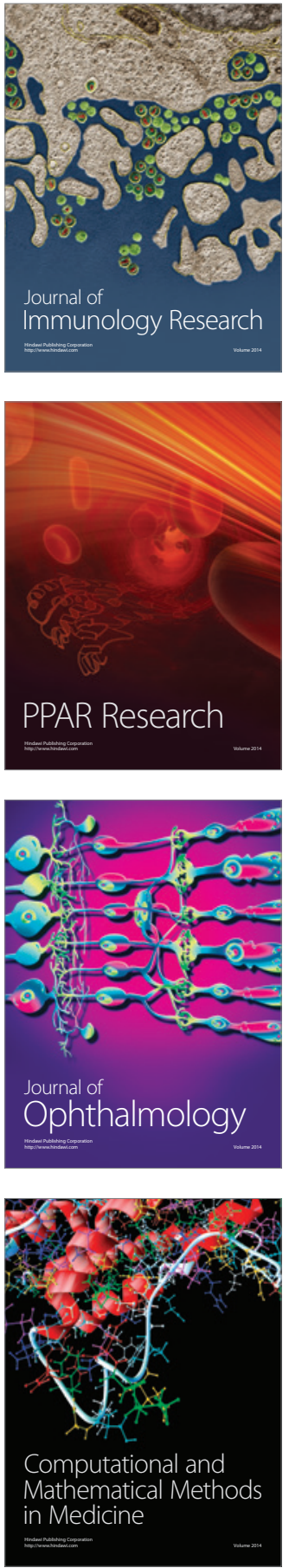

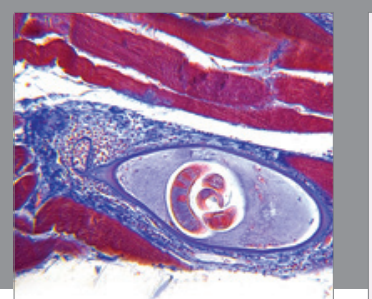

Gastroenterology Research and Practice

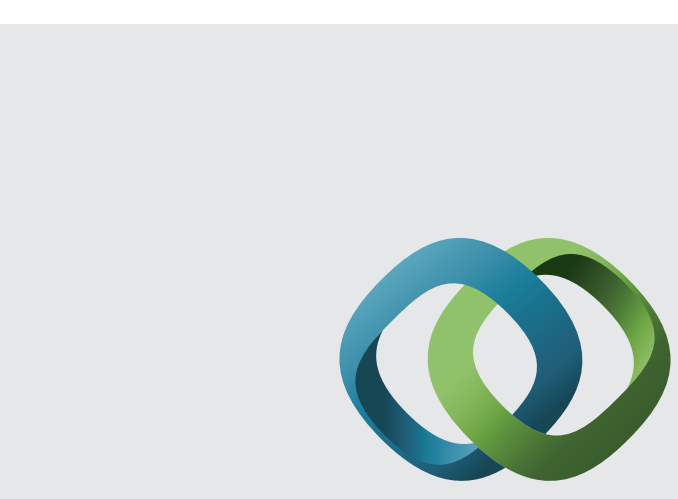

\section{Hindawi}

Submit your manuscripts at

http://www.hindawi.com
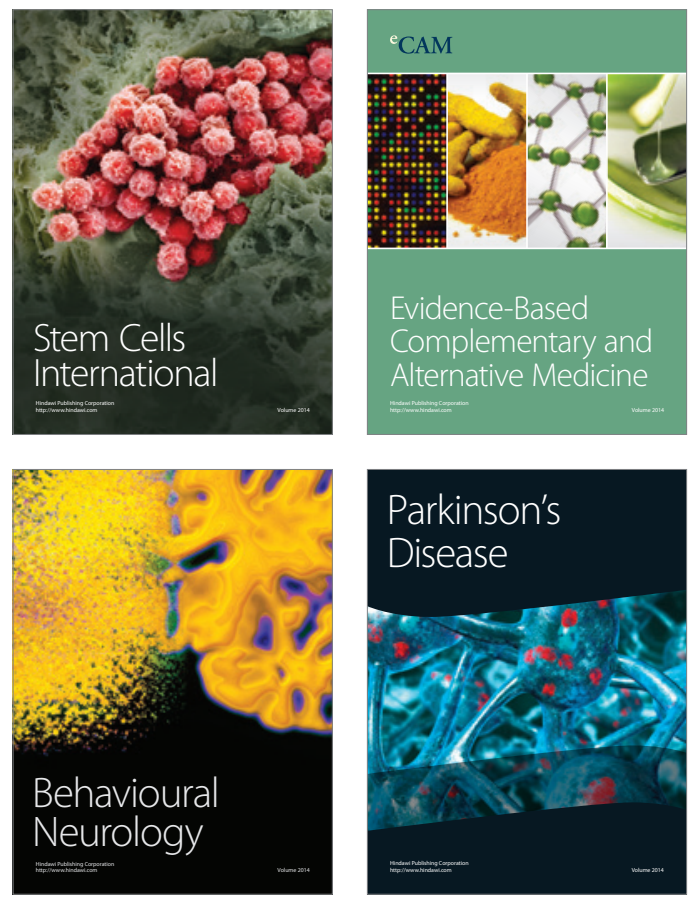
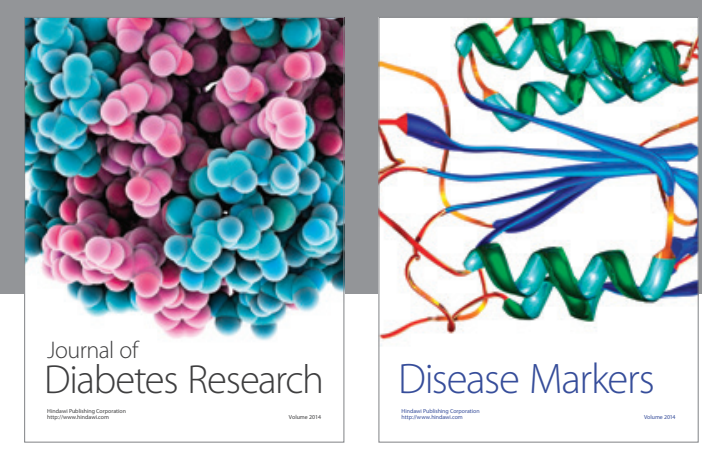

Disease Markers
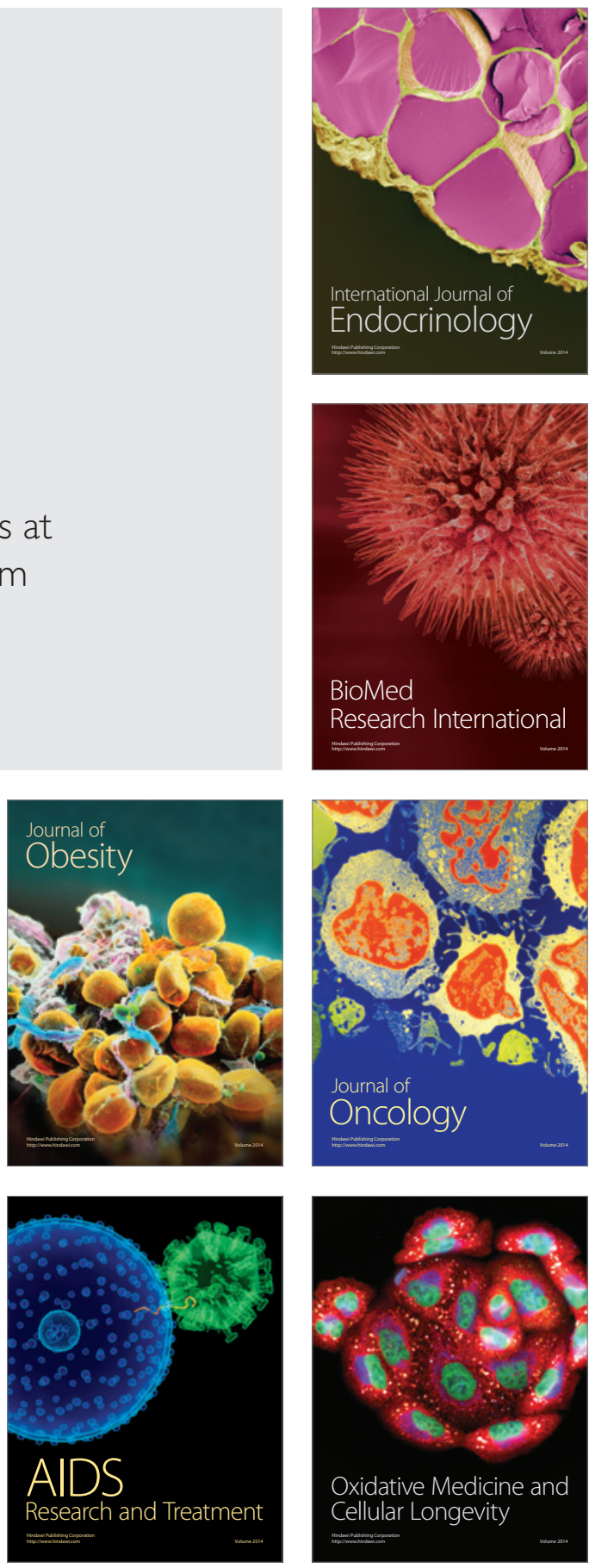